\title{
Impulse excitation measurement of small changes in elastic moduli and damping using $\mathbf{R}$
}

D. Ridley-Ellis ${ }^{\mathrm{a} *}$, M. Libeau ${ }^{\mathrm{b}}$ and D. Mignerat ${ }^{\mathrm{c}}$

${ }^{a}$ Centre for Wood Science \& Technology, Edinburgh Napier University, Edinburgh, UK

${ }^{b}$ Centre for Wood Science \& Technology, Edinburgh Napier University, Edinburgh, UK \& Bordeaux Sciences Agro, Bordeaux, France

${ }^{c}$ Centre for Wood Science \& Technology, Edinburgh Napier University, Edinburgh, UK \& Institut polytechnique de Grenoble, Grenoble, France

* Institute for Sustainable Construction

Edinburgh Napier University

Unit 1, 7 Hills Business Park, 37 Bankhead Crossway South, Sighthill

Edinburgh EH11 4EP

$+44(0) 1314552449$

d.ridleyellis@ napier.ac.uk 
Impulse excitation measurement of small changes in elastic moduli and damping using $\mathrm{R}$

Impulse excitation is commonly used to assess wood stiffness in both scientific and industrial settings. Modes of vibration are excited by a mechanical impulse and their resonant frequencies measured to calculate elastic moduli. It is also possible to assess damping, which is an important property for wood used in musical instruments. This paper covers finer details of the signal analysis necessary when making very accurate measurements. Provided code for Open Source statistical software $\mathrm{R}$ is illustrated with data from an experiment tracking small changes in Douglas-fir clear wood during thermal treatment. A frequency domain interpolation method is introduced that enhances the potential precision of the technique to a very high degree, with computational efficiency, even when using inexpensive audio equipment. With multiple measurements, resonant frequencies can be assessed to a precision of $0.1 \mathrm{~Hz}$ and better, allowing for tracking of very small changes due to wood treatments or moisture content.

Keywords: Non-destructive testing; acoustics; resonance; vibration; heat treatment; open source; wood quality

\section{Introduction}

\section{Outline}

This paper discusses the use of free Open Source statistical computing software R, for the processing of sound recordings made for impulse excitation measurement of wood specimens, and code is made available for other users. Impulse excitation is a very well established non-destructive testing technique that is very useful, but not greatly used, for studying small changes in stiffness and damping due to a wood treatment or modification. The technique is widely employed, and straightforward, for wood characterisation and grading, but here the fine details of signal analysis, required for monitoring of very small changes, are briefly discussed. A spline based frequency 
domain interpolation technique is introduced that allows very precise measurements, which are quick to make, without being computationally demanding. Precision of 0.1 $\mathrm{Hz}$ and better can be achieved with an inexpensive consumer microphone.

The use of the technique, and algorithms, is illustrated with a small selection of data from an experiment tracking small changes in modulus of elasticity, and damping, on Douglas-fir clear wood specimens during heat treatment at $130 \mathrm{C}$.

\section{The impulse excitation method}

Impulse excitation (also known by names such as impact excitation, resonance, vibration, eigen frequency and ping test) is a non-destructive material characterisation technique in which an elastic solid is lightly struck with a hammer or projectile, giving it energy to vibrate at its natural frequencies. The induced vibration response, resulting from this momentary impulse, is measured with a suitable transducer such as a microphone, piezoelectric sensor, laser vibrometer or accelerometer. The acquired vibration signal in the time domain is converted to the frequency domain to determine the modal frequencies. If the shape is suitable (e.g. rectangular bars, cylindrical rods) these frequency spectra peaks can be used with geometry and mass measurements to calculate Young's modulus, shear modulus, Poisson's ratio and internal friction. The technique is covered by several standards (e.g. ASTM E1876, ISO 12680-1, EN 843-2 and EN 14146), but, with the exception of the ASTM D6874-12 not yet one specifically for wood, which is notable for its anisotropy and consequently more complex relationship between elastic and shear moduli. It is, nevertheless, widely used in wood science and commercial timber production and several strength grading machines use this principle in its most straightforward form.

The test specimen is encouraged to vibrate in certain target modes by the way it is shaped, supported and struck, and these modal frequencies emphasised in the 
recording by where and how the response is measured. The anisotropy and large range of plausible stiffness of wood can sometimes make matching of peak frequencies with modes challenging, but this can be resolved by playing resonant frequencies at the specimen (continuous excitation) and observing Chladni patterns, or a technique such as Very Near Field (Pantelić et al. 2017).

The anisotropic and inhomogeneous nature of wood also means that the standard equations for materials like metals and ceramics might not necessarily give a correct value of the calculated stiffness property, and more sophisticated numerical models may be required. This is especially true where the specimens are not slender bars. If the grain is oriented along the specimen length, the anisotropy itself is not a source of substantial error. However error may come about by an inappropriate assumption, of how shear modulus relates to modulus of elasticity, within the formulation of the equation (see Brancheriau and Bailleres 2002 for a theoretical review relevant to wood). However, if the aim is to monitor changes in stiffness due to a treatment of the sample it may be adequate to use an approximate equation, and percentage change in stiffness rather than absolute values. There is also the problem of adjusting the 'dynamic' value of the property to the 'static' value representative of what would be measured in a standard mechanical test such as three or four-point bending. These important matters, discussed in many other places, are out of the scope of this short paper, which instead concentrates on the step of processing the acquired vibration signal in a fast, convenient, reliable and accurate way to determine modal frequencies and damping.

In practice, the acquired vibration signal is commonly processed with a dedicated software package, the instrument electronics, or with software for general signal analysis. In those cases, the algorithms applied are not usually revealed to the user and errors can be made as a consequence. Gross errors are easy to spot, but small 
errors are not and these may be significant when there is a need for precise and accurate measurement of very small changes in wood properties following some treatment process, especially when this also affects dimensions and mass.

Quantities measured: frequency f and damping decay constant $\lambda$ (or bandwidth quality factor $Q$ )

The basis of the model is the theoretical, perfect, response signal of a single mode (underdamped harmonic vibration) described mathematically by the standard harmonic motion Equation 1. Where there are several modes, the response is the sum of the component exponentially decaying sine waves for each. The frequency, as assessed by the peak on an FFT plot $\left(f_{\text {peak }}\right)$, can be corrected by equations 2 and 3. This adjustment is small but may be necessary for high precision measurement.

$$
\begin{aligned}
& A_{t}=A_{\text {max }} \times e^{-\lambda t} \times \sin (2 \pi f t) \\
& \lambda=\pi B \approx \pi f_{\text {peak }} / Q \\
& f=\sqrt{f_{\text {peak }}^{2}+(\lambda / 2 \pi)^{2}}
\end{aligned}
$$

$t$ is the time, starting at zero when the wave begins

$A_{t}$ is the amplitude at time $t$

$A_{\max }$ is the initial amplitude of the envelope

$B$ is the $-3 \mathrm{~dB}$ bandwidth $(\mathrm{Hz})$

$f$ is the natural frequency $(\mathrm{Hz})$

$f_{\text {peak }}$ is the peak frequency in the FFT $(\mathrm{Hz})$

$\lambda$ is the exponential decay constant $\left(\mathrm{s}^{-1}\right)$ 
$Q$ is the bandwidth quality factor

\section{Software used}

The software used for this paper is R ( $\mathrm{R}$ Core Team, 2017), a language and environment for statistical computing and graphics. Although primarily intended for statistics, it has much wider uses and, being free and Open Source, has the advantage that all calculation algorithms are open to inspection by the user, and by others. The R user community provides many packages that add extra functions to the base $\mathrm{R}$ code. In this paper the method uses the packages Seewave (created for bioacoustics analysis) (Sueur et al., 2008) and tuneR (created for analysis of music and speech) (Ligges et al., 2016). Several new functions have been written, specifically tailored to impact excitation on wood. The set of R scripts (referred to here as "TimbR") are made available online (http://blogs.napier.ac.uk/cwst/iet/) and others are invited to comment on, improve and extend the code for the benefit of wood scientists and engineers generally. By openly showing the algorithms it is hoped that users of dedicated equipment and software for impact excitation are more informed as to what kind of calculations are being done. The algorithms can also be adapted for use in other environments such as MATLAB. It is not intended to replace any specialist software or equipment (such as the Bing system by Cirad, intended specifically for use with wood, the Buzz-o-Sonic system from BuzzMac, and the long in use GrindoSonic from JW Lemmens), but rather as a learning tool, and entry level option for new and occasional users of the impulse excitation technique, such as researchers of wood modification. 


\section{Procedure and algorithms}

\section{Making measurements}

The impact excitation method is easily used on sawn timber and small clear wood samples, is fast and reliable, and need not necessarily require the use of expensive specialist equipment. If the important frequencies are in the range of human hearing (commonly the case with wood), and the measurement environment reasonably quiet, accurate measurements can be obtained using only a basic consumer microphone. Indeed, the measurement of frequency response is less demanding than the other necessary measurements of mass, dimensions and moisture content. It does, however, require some good measurement technique, especially for measurement of damping.

There are several possible vibration modes, especially when the specimen is plate-like in shape. This means that there are often many resonant frequencies present in the recording, and sometimes frequencies for modes coincide. The most useful modes of free-free vibration (Fig. 1) useful for rectangular sawn timber are:

- Longitudinal - a simple vibration mode (and harmonics) that is the basis of measurement of several timber grading machines including the Dynalyse Dynagrade and Precigrader, the MiCROTEC Viscan series, the Brookhuis MTG series and similar tools for logs like the fibre-gen HITMAN HM200 and LG640.

- Flexural (major, 'in plane' or minor axis, 'out of plane') - which is more complicated to analyse, more sensitive to cross-section dimensions, grain orientation and anisotropy. It is the basis of measurement for timber grading machines such as the SARL Esteves Noesys and XYLOMECA Xyloclass F, is the type of mode more commonly used for the impact excitation technique on 
other materials, and the most relevant mode for measurement for tonewood for musical instruments.

- Torsional - a twisting vibration mode (and harmonics) that can be used to assess shear modulus.

When calculating stiffness from the measured frequency, density and dimensions, it is necessary to use equations appropriate for the support conditions, shape and aspect ratios. When a specimen is resting on supports which do not impede its movement for the target mode, its vibration is 'free-free' (i.e. the supports do not determine the node positions).

Note that for specimens with poor aspect ratio (and for higher order modes), the theoretical correction factors given in equations usually assume isotropic material properties, and therefore an implicit relationship between shear modulus and longitudinal elastic modulus that is inaccurate for wood. This is also true of the shear correction (shape) factor from Timoshenko's beam theory that appears in the flexural vibration equation (see Brancheriau 2006). The different shear modulus in radiallongitudinal and radial-tangential planes also adds complexity not often covered by commonly used equations. If there is doubt, modal analysis with finite element method and anisotropy can be used. For convenience, equations suitable for wood, for the three mode types of vibration, (with references) are provided within the TimbR set of functions.

$\mathrm{R}$ is capable of recording audio but here it is assumed that this has been done already and that there is an audio file containing reasonably clean recordings for several repeated measurements ('hits') on a specimen. For best results, the audio should be recorded and saved without any kind of lossy compression, since compression 
algorithms subtly change the frequency spectrum. The recording device should not continuously adjust the recording level as this will affect the measurement of damping.

For the TimbR R scripts presented in this paper, it is assumed that audio is saved as pulse-code modulation waveform audio file format, that these wav files are mono (or that stereo information is unimportant), and that the file header contains the sampling rate and bit depth. Knowing the sampling rate of recording is vital and an error made in this respect can be very hard to spot in the results (especially between common rates 44.1 and $48 \mathrm{kHz}$ ). The R script can open a directory of wav files, each with multiple hits, and work through all of them automatically.

There are several ways in which a recording can be imperfect, including: too long hammer contact duration, accidental double strike, signal overload, recording starting too late or ending too soon, a subsequent impact being made before the vibration response has subsided, background noise, and movement of the microphone. Good measurement discipline can minimise these, but the signal analysis should be robust against them and notify the user where there might be problems so the signal can be manually inspected, or the results discarded. One checking procedure is implemented by way of the waveforms of the strikes being reconstructed from the calculated peak frequency and damping for playback, allowing incorrect data to be easily heard as unexpected sound.

\section{Algorithm for detecting hits and applying the window function}

On opening a sound file, the first step is to remove any zero offset that might be present by subtracting, from the wave, the mean of all amplitude values. Following this, the wave is examined to locate the start and end of each hit recoding. Seewave contains a function ('timer') that can do this, but it requires some user tweaking of thresholds and time adjustment to get the start and end points correct. So instead a new function is 
written that makes use of the special circumstances for impact excitation (knowing that the signal starts with a high amplitude and decays towards silence).

Fast Fourier Transform (FFT) assumes that the signal is periodic, which is true of impact excitation if the recording begins and ends with silence. True silence is unlikely in reality so a windowing function is useful to create this condition, reducing the effects of 'leakage' (a problem for damping calculation). An exponential window is appropriate for impact excitation, and it is simple to correct the calculated damping to remove the additional damping effect of the window. A new $\mathrm{R}$ function is provided that automatically applies an appropriate exponential window and corrects results accordingly.

\section{Algorithm for detecting peaks and calculating damping}

The wav segments for each hit are analysed with a function based on the ' $\mathrm{fft}$ ' function in base (stats) R. The frequency spectrum is then analysed to find the frequency peaks. Seewave provides the function 'fpeaks', but this requires user configuration, is slow to calculate, and was found to be not good at distinguishing between genuine impulse excitation peaks and noise. A new algorithm is provided which is considerably faster, and also calculates damping (through bandwidth Quality Factor, Q) at the same time.

Impact excitation is commonly done based on simple accept/reject criteria, where a measurement is accepted if the impact seems good and the same frequency has been obtained a few times in a row. Since each hit is similar duration, the FFT frequency bins are the same each time meaning that the peak frequency can only take certain discrete values, limiting the usefulness of taking averages of multiple measurements. Zero padding of the signal can be used to decrease the size of the FFT bins, but this becomes very computationally expensive as desired precision increases. 
This paper introduces a way of extracting additional precision and confidence from repeat measurements by using spline curve interpolation at points of interest in the frequency domain. Interpolation methods in the frequency domain for FFT are certainly not new in other fields, but this implementation is. The spine goes though all frequency points within the region for calculating peak frequency (Fig. 2) and bandwidth Q improving the precision of calculation of both, so long as multiple repeat measurements are made and the specimen response does not change between them. The R script uses a combination of zero padding and spline interpolation to obtain precision with good computation speed. Note that this approach does not provide extra precision that would allow differentiation of two closely positioned peaks, and in such cases it is better to emphasise the desired peak through the way the impact excitation is done.

A function is also provided for calculating damping from the logarithmic decrement in the time domain, although this is rather difficult to do accurately unless all undesired modes can be reduced (since the modes have different damping). When more than one mode is present the signal requires to be first filtered around the frequency of interest.

\section{Testing the performance with simulated recordings}

The performance of the algorithms, including the spline interpolation, was tested by analysing perfect (mathematically generated) signal data with a single frequency component. For each simulated signal length shown here, there are 2000 simulated signals with properties drawn randomly from uniform ranges of damped frequency between $20 \mathrm{~Hz}$ and $20 \mathrm{kHz}$, and damping decay rates between 5 to $50 \mathrm{~s}^{-1}$.

Since the frequency and damping are known exactly, they can be compared to the values calculated from the signal analysis, both with and without spline 
interpolation. An exponential window is applied in the analysis to ensure signals are adequately decayed within the measurement length (a window decay rate between $3.4 \mathrm{~s}^{-1}$ and $69 \mathrm{~s}^{-1}$ ). A small amount of zero padding is applied (up to $0.01 \mathrm{~s}$ ) to increase the number of factors for the time domain points, and thereby increase FFT calculation speed and reduce memory usage.

Fig. 3 shows the calculation accuracy for peak frequency (closest FFT bin vs. interpolation). Errors are expressed as the $5^{\text {th }}$ percentile of over and underestimation (i.e. $90 \%$ of errors fall between these extremes). As expected, the frequency error when simply taking the FFT bin with the highest amplitude is symmetrical, uniformly distributed, and as wide as an FFT bin. The use of spline interpolation was seen to improve frequency precision by a factor of about 10 (or better) compared to simply taking the FFT bin with the highest amplitude. For 0.4 second recording duration, the factor is 20 and frequency precision is $\pm 0.5 \mathrm{~Hz}$ across the range of frequencies. Analysis is least accurate when waves have very low $Q$ factors.

Fig. 4 shows the comparison for damping (linear interpolation from FFT for bandwidth, vs. spline interpolation). Spline interpolation performs better, reducing the error by a factor of 2 or better ( 4 or 5 for shorter recordings). Provided the recording is long enough ( 0.3 seconds), the damping decay rate measurement accuracy is better than $\pm 0.5 \mathrm{~s}^{-1}$. Failure to properly window a recording tends to cause much larger errors in the assessment of damping.

These simulated recordings were 16-bit $44.1 \mathrm{kHz}$ wav. Bit depth is unimportant for the simulation, but in practice, extra bit depth allows quieter strikes to be adequately captured. The use of a higher sampling rate would allow measurement of higher frequencies, but not provide increased precision of frequencies since the FFT bin width 
is not reduced. While precisions for frequency and damping can also be increased by zero padding of the time domain, this is very much slower for calculation.

\section{Illustration with real data}

Real recordings are more challenging than perfect mathematical signals, containing experimental measurement error and noise. Figs. 5 and 6 are a brief illustration of the results of TimbR analysis of impact excitation recordings made on four clear-wood specimens (named G5, G6, G7 and G8), at different points during a heat treatment experiment. During the treatment there are small changes in elastic properties, mass and dimensions which result in small changes in frequency response between these measurement points. The technique and analysis is accurate enough to detect these changes and track them during the experiment.

The four specimens were Douglas-fir (Pseudotsuga menziesii (Mirb.) Franco.) of nominal dimensions $300 \mathrm{~mm}$ (longitudinal $) \times 68 \mathrm{~mm}($ radial $) \times 18 \mathrm{~mm}$ (tangential) cut from a single board (original density $580 \mathrm{~kg} / \mathrm{m}^{3}$ at $12 \% \mathrm{mc}$ ). The recordings were made with a USB computer microphone at $48 \mathrm{kHz}$ and 16 bit depth. These specimens were being heated in an oven at $130 \mathrm{C}$ (in air), removed from the oven, allowed to cool in indoor air (no desiccator) for impulse excitation measurement (at which time the moisture content was approximately $0.2 \%$ ) and then returned to the oven. The small subset of the results shown here are for the first mode of flexural minor axis (out of plane, Fig. 1b) vibration. The specimens were supported by two threads at about $65 \mathrm{~mm}$ from the ends, and impacts made manually with a hammer with spherical polymer tip. Results are presented here for the period between 50 and 150 days, which is enough to show the repeatability and precision of the approach. In this particular example, the change in frequency is easily observable. About 20 hits are made on each specimen at each measurement point, for which measured frequency varied by about $\pm 0.2 \mathrm{~Hz}$. The 
change of approximately $10 \mathrm{~Hz}$ between these measurement points shown equates to a decrease in elastic modulus of about $2.5 \%(\sim 10 \%$ overall during this treatment period Fig. 7). For elastic modulus, the much larger source of error is now the measurement of the thickness of the specimen, which is complicated in this case by the different shrinkage of the earlywood and latewood as the heat degraded the wood.

The change (if any) in damping is less clear. The analysis is reasonably repeatable and is capable of measuring a change, but if any exists in this case it is obscured by uncontrolled measurement parameters in this particular experiment, which was not initially intended for assessment of damping (such as exact support condition, strength of hammer strike and slight thermal or moisture gradient in the specimen).

\section{Conclusions}

This paper showed some frequency analysis performance tests on an open source $\mathrm{R}$ script for impact excitation measurement, built on the packages Seewave and tuneR, and with several new algorithms for impulse excitation. The script aims to be as automatic as possible, requiring little configuration by the user, while also being completely transparent about the mathematical procedures, and readily customised.

Specifically, interpolation methods are introduced that enhance the precision of the technique to a very high degree when making multiple repeated measurements. Resonant frequencies can potentially be assessed to a precision of $0.1 \mathrm{~Hz}$ and better, allowing for tracking of very small changes due to wood treatments or moisture content. Calculation of damping by bandwidth method is also improved, subject to proper measurement practice.

Other researchers are invited to improve and build on this $\mathrm{R}$ code and its methods. It is not presented as a replacement for measurement by specialist equipment such as Grindosconic, BING, and Buzz-o-sonic, or the self-built systems in use in some 
research laboratories, but rather to assist new and occasional users (such as wood modification researchers) in understanding how the measurements are processed, what errors may result if used incorrectly, and as an entry-level option for employing the technique, with high precision capability.

\section{References}

ASTM D6874-12, Standard Test Methods for Nondestructive Evaluation of WoodBased Flexural Members Using Transverse Vibration, ASTM International, West Conshohocken, PA, 2012, www.astm.org

ASTM E1876-15. 2015. Standard Test Method for Dynamic Young's Modulus, Shear Modulus, and Poisson's Ratio by Impulse Excitation of Vibration, ASTM International, West Conshohocken, PA, http://www.astm.org

Brancheriau L. 2006. Influence of cross section dimensions on Timoshenko's shear factor - Application to wooden beams in free-free flexural vibration. Annals of Forest Science 63 (3):319-321.

Brancheriau L, Bailleres H. 2002. Natural vibration analysis of clear wooden beams: a theoretical review. Wood Science and Technology 36:347-364.

EN 843-2:2006 Advanced technical ceramics. Mechanical properties of monolithic ceramics at room temperature. Determination of Young's modulus, shear modulus and Poisson's ratio, CEN, Rue de la Science 23, B - 1040 Brussels, Belgium. https://www.cen.eu

EN 14146:2004 Natural stone test methods. Determination of the dynamic elastic modulus of elasticity (by measuring the fundamental resonance frequency), CEN, Rue de la Science 23, B - 1040 Brussels, Belgium. https://www.cen.eu

ISO 12680-1:2005 - Methods of test for refractory products -- Part 1: Determination of dynamic Young's modulus (MOE) by impulse excitation of vibration. ISO, Geneva, Switzerland. http://www.iso.org

Ligges U, Krey S, Mersmann O, Schnackenberg S. 2016. tuneR: Analysis of music. http://r-forge.r-project.org/projects/tuner/

Pantelić F, Ridley-Ellis D, Mijić M, Šumarac Pavlović D. 2017. Monitoring changes in wood properties using Very Near Field sound pressure scanning. In: Proceedings of the COST FP1302 WoodMusiCK conference; Oct 5-7; Brussels.

R Core Team, 2017. R: A Language and Environment for Statistical Computing, R Foundation for Statistical Computing (v3.4.1), Vienna, Austria, http://www.Rproject.org 
Sueur J, Aubin T, Simonis C. 2008. Seewave: a free modular tool for sound analysis and synthesis. Bioacoustics 18:213-226. http://rug.mnhn.fr/seewave/ 


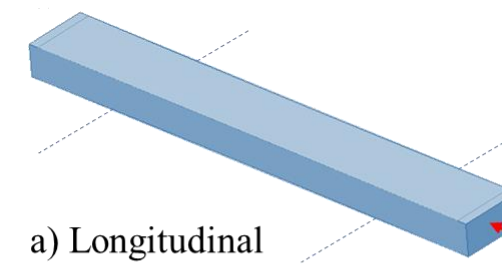

a) Longitudinal

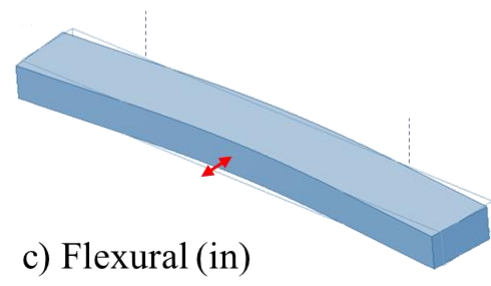

b) Flexural (out)

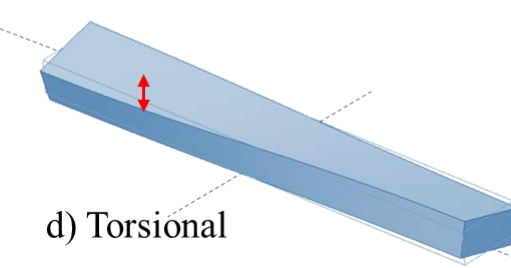

Figure 1. Useful modes of vibration for impact excitation

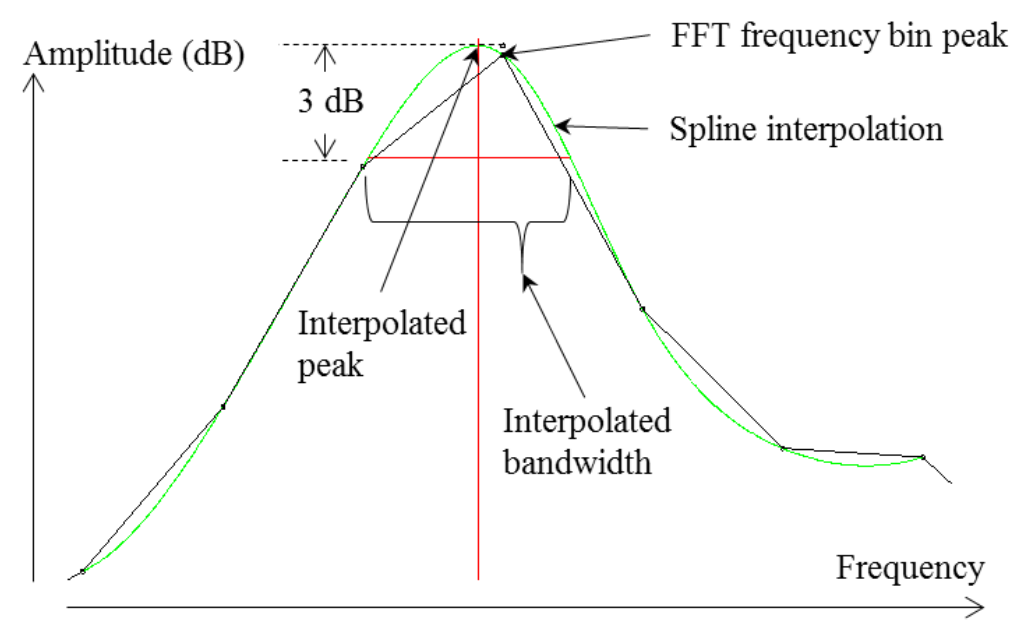

Figure 2. Spline interpolation for the peak finding algorithm 


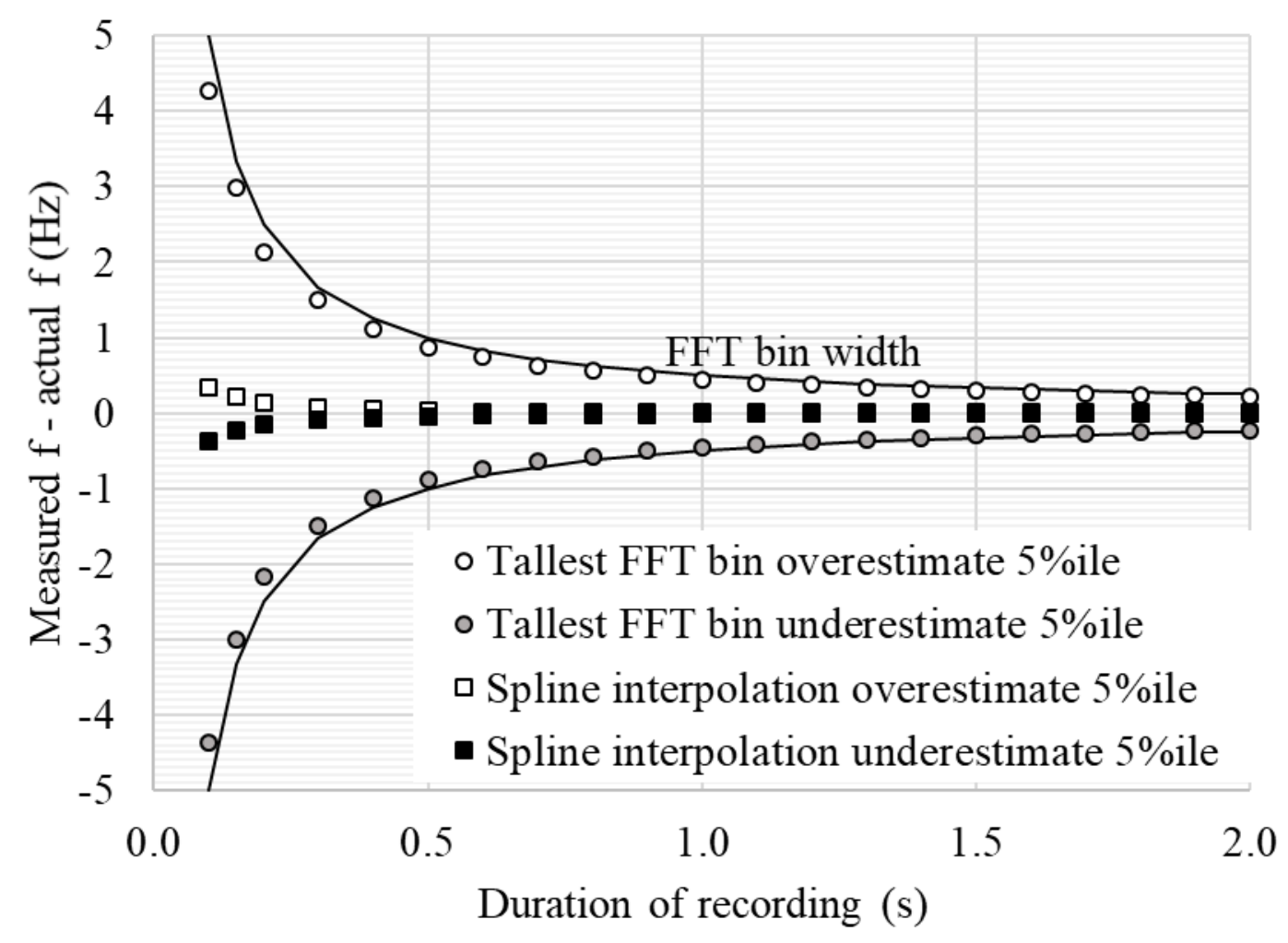

Figure 3. Accuracy for frequency (within $20 \leq f \leq 20000 \mathrm{~Hz}$ and $5 \leq \lambda \leq 50 \mathrm{~s}^{-1}$ )

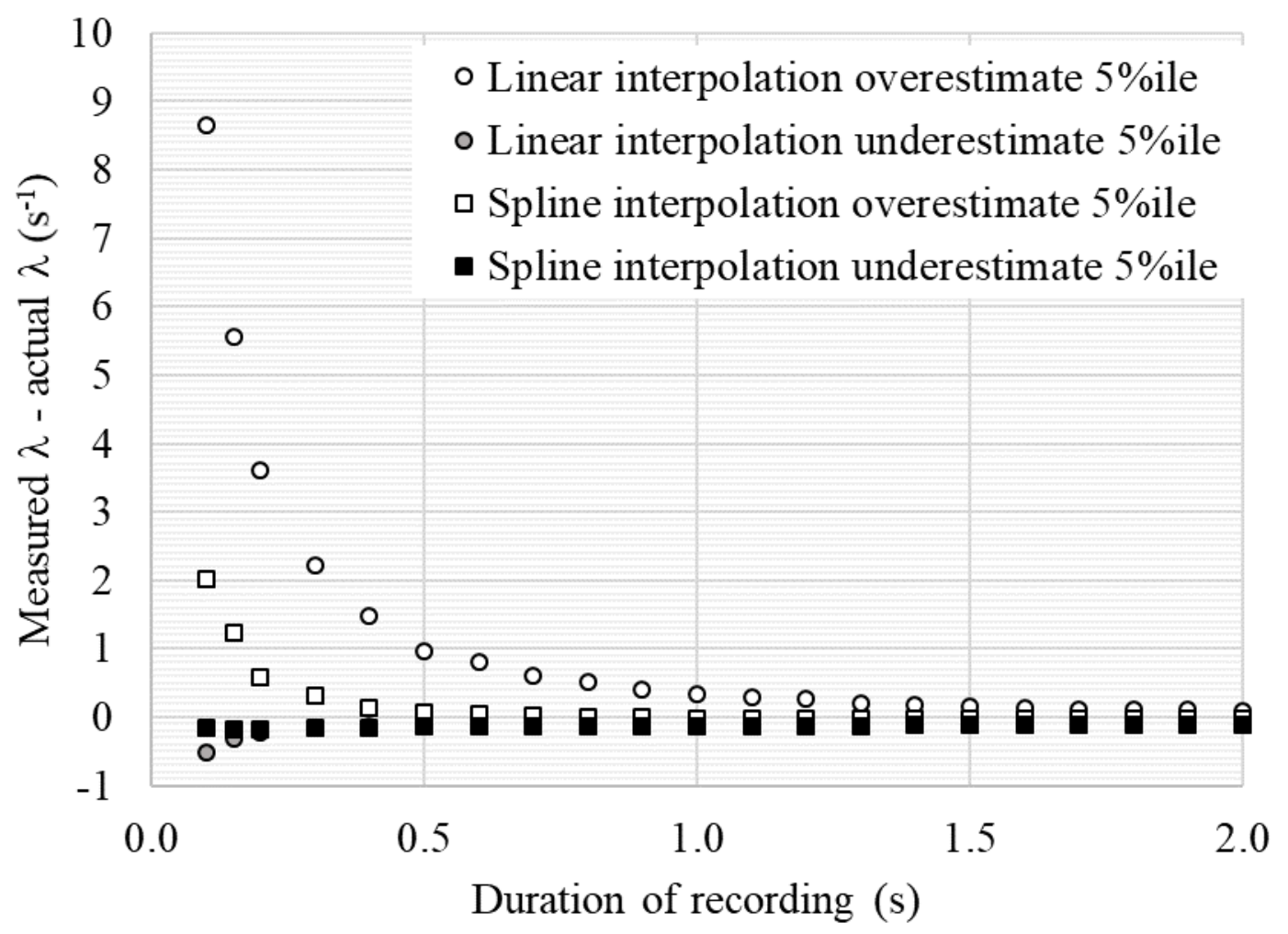

Figure 4. Accuracy for decay rate $\lambda$ (within $20 \leq f \leq 20000 \mathrm{~Hz}$ and $5 \leq \lambda \leq 50 \mathrm{~s}^{-1}$ ) 


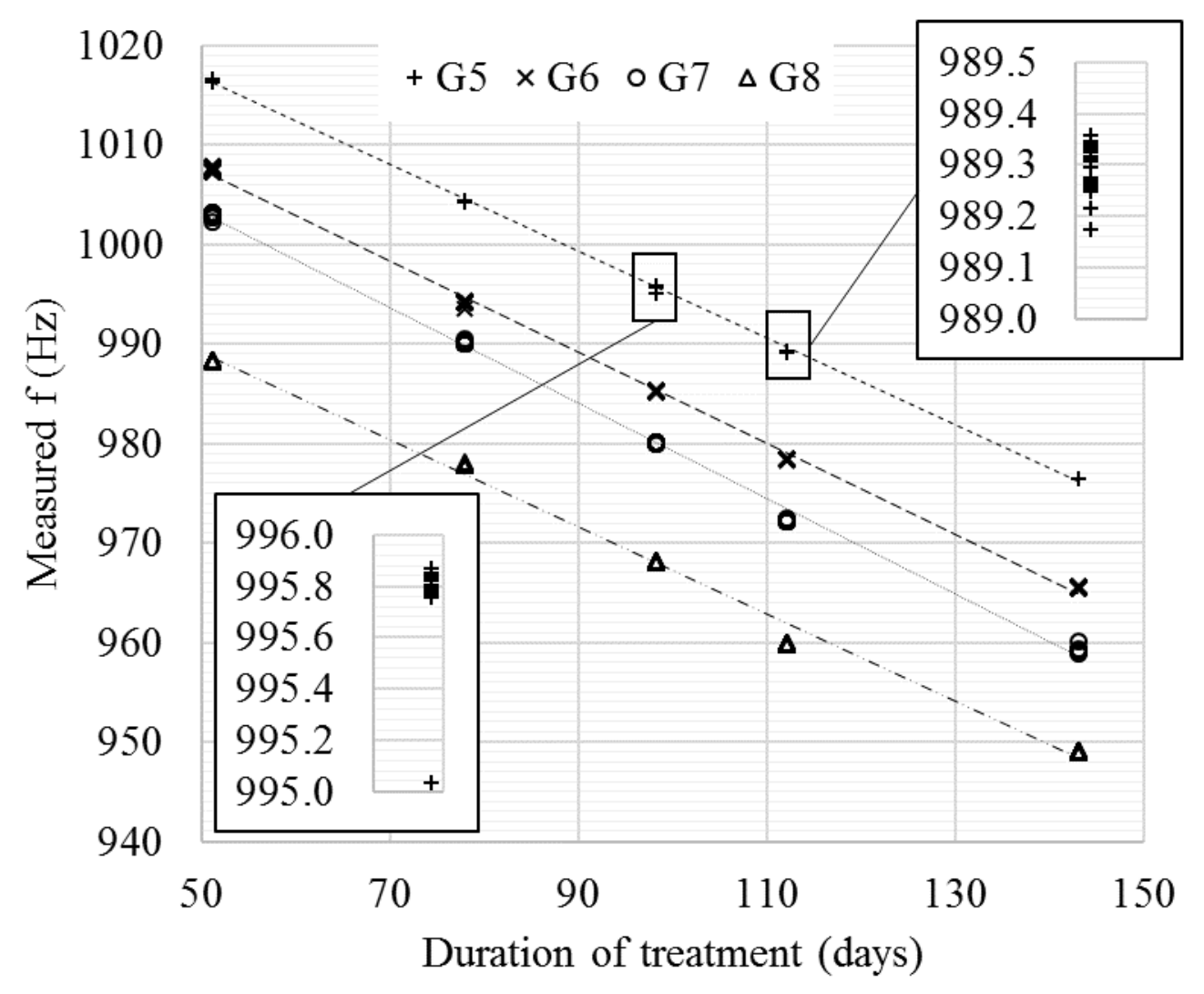

Figure 5. Measurements of frequency f for first flexural mode, during heat treatment

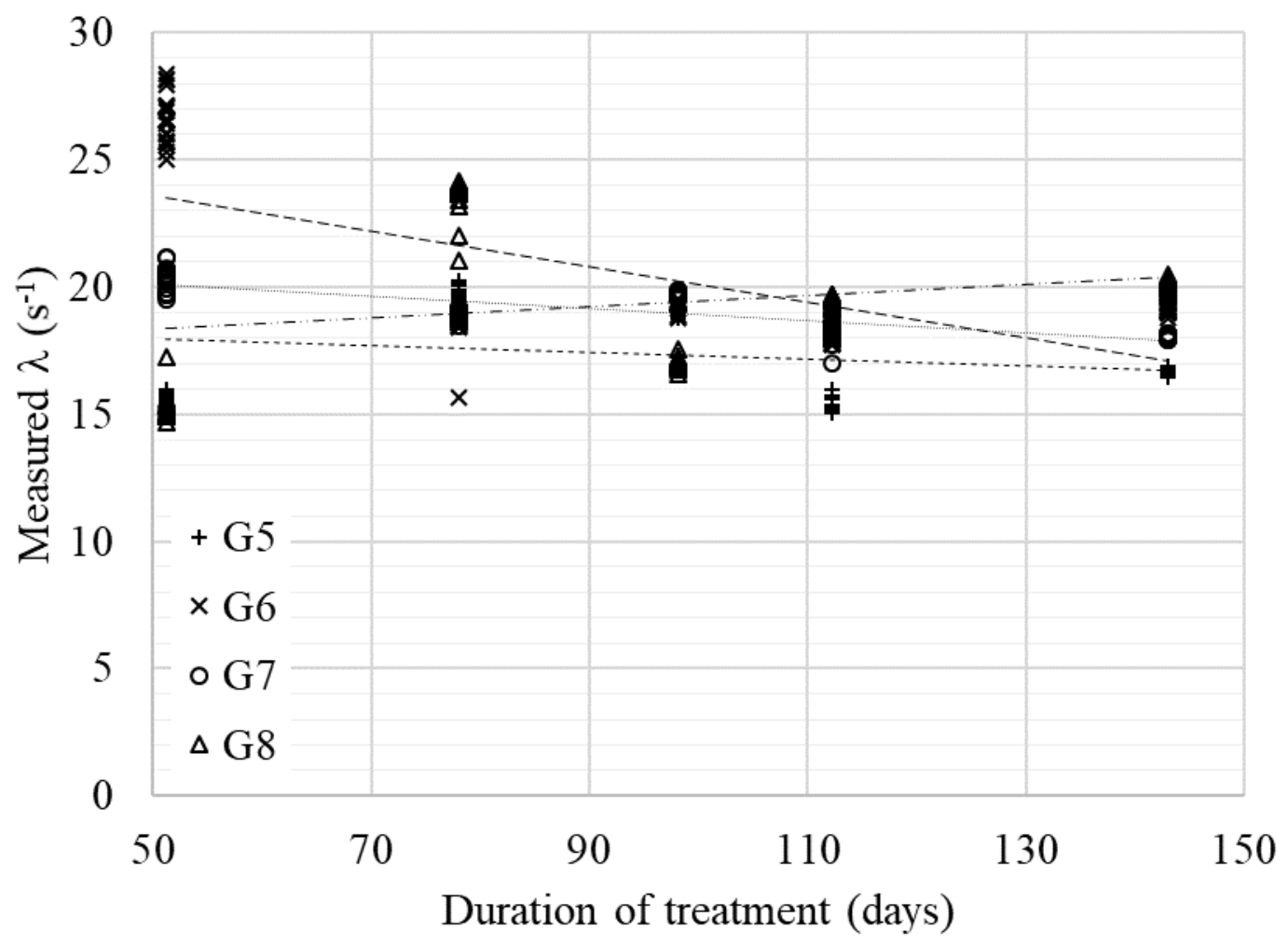

Figure 6. Measurements of decay rate $\lambda$ for first flexural mode, during heat treatment 


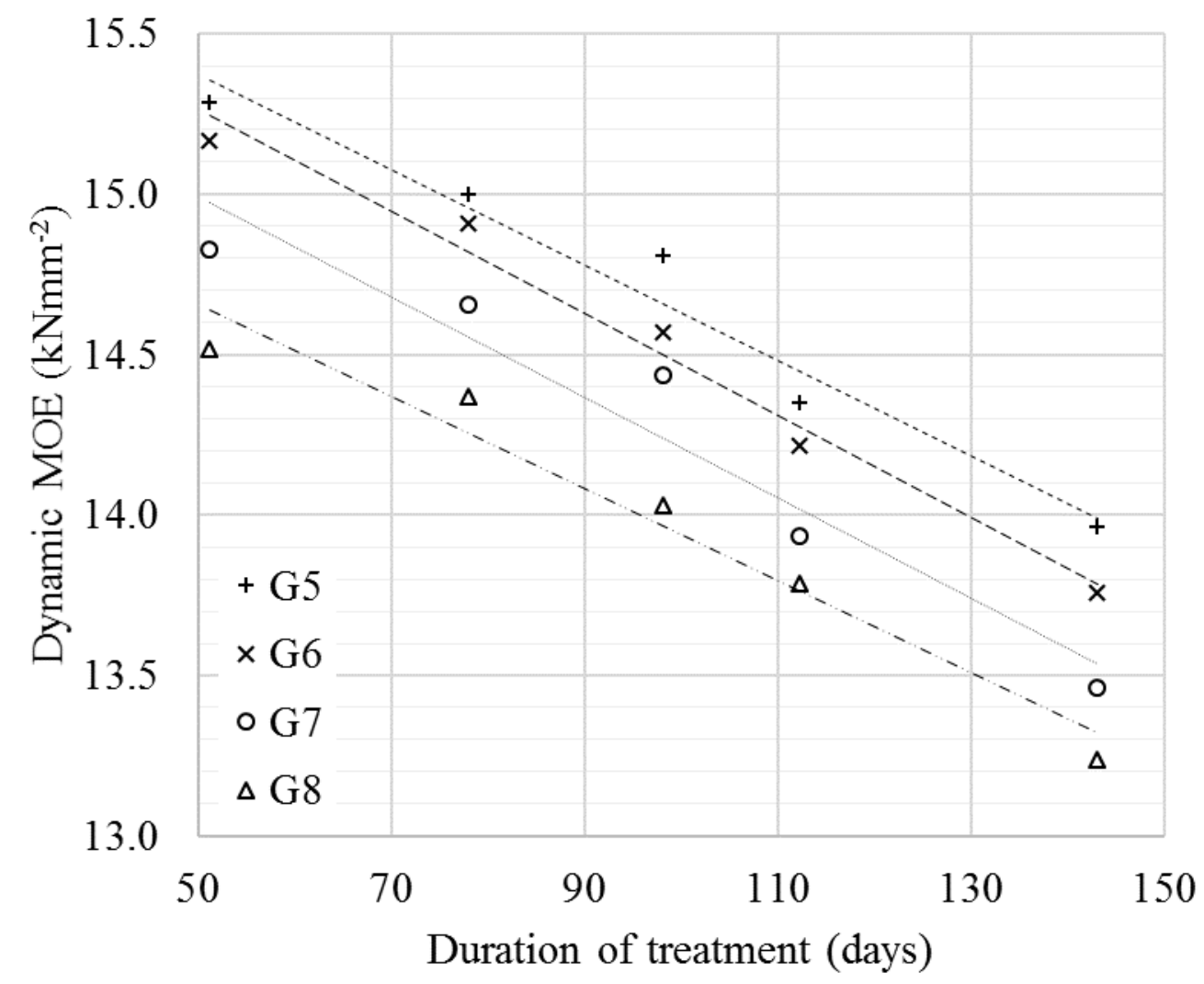

Figure 7. Change in dynamic modulus of elasticity, estimated from first flexural mode, during heat treatment 